\author{
РАЗРАБОТКА БОРТОПОВОРОТНОГО ШАССИ \\ И ВЫБОР СИСТЕМЫ НАВИГАЦИИ \\ ДЛЯ ЭКСПЕРИМЕНТАЛЬНОЙ МОДЕЛИ МОБИЛЬНОГО РОБОТА \\ Горшунов А.А., Кузьменко С.В., Сергеев Е.И., Ходосов Е.С., \\ Татаринов П.С., Ким Д.Ч., Семёнов А.С. \\ Северо-Восточный федеральный университет имени М.К. Аммосова, \\ Политехнический институт (филиал), Мирный, e-mail: sash-alex@yandex.ru
}

\begin{abstract}
Статья посвящена актуальной теме исследований, связанной с проектированием и разработкой робототехнических устройств и систем их навигации. В рамках работы студенческого научного кружка её авторами было разработано бортоповоротное шасси экспериментальной модели мобильного робота-уборщика. Подробно описана конструкция и представлена кинематическая схема шасси. В качестве электропривода выбран двигатель постоянного тока из-за простоты регулирования его оборотов. Для управления оборотами двигателя постоянного тока применена широтно-импульсная модуляция. На практике ШИМ регулятор был реализован с помощью микроконтроллера Arduino Uno. В программной среде Arduino IDE была разработана программа автоматического управления скоростью вращения двигателя по желаемому графику. В результате анализа и теоретических исследований в качестве навигационной системы робота были выбраны два метода: метод отражённой волны и инерциальный метод. Представлены преимущества и недостатки каждого из них. Разработана установка волнового зондирования с добавлением инерциальной составляющей в модель. Описаны преимущества такой совмещенной системы навигации. Также отмечены главные недостатки, решить которые помогла разработка принципиально новой интегрированной системы инерциально-зондовой навигации, в которой был применен более мощный микроконтроллер Arduino Mega. Сделаны заключения о значимости исследований и о возможных продолжениях реализации проекта по созданию экспериментальной модели мобильного робота.
\end{abstract}

Ключевые слова: робототехника, мобильный робот, бортоповоротное шасси, система навигации, микроконтроллер, Arduino

\title{
DEVELOPMENT OF THE BATTOM CHASSIS AND SELECTION OF THE NAVIGATION SYSTEM FOR THE EXPERIMENTAL MODEL OF MOBILE ROBOT
}

\section{Gorshunov A.A., Kuzmenko S.V., Sergeev E.I., Khodosov E.S.,} Tatarinov P.S., Kim D.Ch., Semenov A.S.

North-Eastern Federal University named after M.K. Ammosov, Polytechnic Institute (branch), Mirny,e-mail: sash-alex@yandex.ru

\begin{abstract}
The article is devoted to the actual topic of research related to the design and development of robotic devices and their navigation systems. Within the framework of the work of the student scientific circle, its authors developed a side-turning chassis of the experimental model of a mobile robot cleaner. The construction is described in detail and the kinematic scheme of the chassis is presented. As the electric drive the DC motor is chosen because of the simplicity of its speed control. To control the speed of the DC motor, pulse-width modulation is applied. In practice, the PWM regulator was implemented using the Arduino Uno microcontroller. In the software environment of the Arduino IDE, a program was developed to automatically control the engine's rotation speed according to the desired schedule. As a result of the analysis and theoretical studies, two methods were chosen as the navigation system of the robot: the reflected wave method and the inertial method. The advantages and disadvantages of each of them are presented. A wave-sounding installation with the addition of an inertial component to the model was developed. Advantages of such a combined navigation system are described. Also noted are the main drawbacks, solved by the development of a fundamentally new integrated inertial-navigation navigation system, in which a more powerful microcontroller Arduino Mega was used. Conclusions are made about the importance of research and about the possible continuation of the project to create an experimental mobile robot model.
\end{abstract}

Keywords: robotics, mobile robot, side-chassis, navigation system, microcontroller, Arduino

Современное высокоточное производство невозможно без использования роботов. Россия в 1990-е гг. утратила свой парк промышленных роботов. Создание роботов и робототехнических систем, предназначенных для автоматизации сложных технологических процессов и операций, в том числе выполняемых в недетерминированных условиях, для замены человека при выполнении тяжёлых, утомительных и опас- ных условиях, является актуальнейшим вызовом нашего времени [1-3].

Студенческий научный кружок (далее СНК) «Робототехника, механотроника и электроника», основанный в 2014 г. и функционирующий на базе кафедры электроэнергетики и автоматизации промышленного производства, в настоящее время занимается разработкой экспериментальной модели мобильного робота, 
который мог бы выполнять операции по уборке территории Политехнического института (филиала) СВФУ им. М.К. Аммосова в г. Мирном.

Предшествующие научные разработки участников СНК основаны на теоретических исследованиях систем управления и навигации робототехнических устройств и подробно изложены в [4-6]. В настоящей статье представлены результаты практических (технических и программных) исследований, позволяющих приступить непосредственно к разработке экспериментальной модели мобильного робота.

Цели и задачи исследования: основными целями исследования являются: разработка колесного бортоповоротного шасси мобильного робота и системы управления им; выбор системы навигации и анализ возможного совмещения различных методов для более точного позиционирования мобильного робота.

Для достижения поставленных целей были решены следующие задачи:

- создание колесного бортоповоротного шасси рабочей модели робота, взаимодействующего с внешней средой;

- выбор системы управления объектом и процессом, определение наилучшего способа управления при заданных целях и критериях;

- исследование и выбор актуальных, рабочих и действенных способов навигации в пространстве;
- создание интегрированной системы навигации, основанной на совместном использовании нескольких методов.

\section{Результаты исследования и их обсуждение}

Бортоповоротное шасси робота конструктивно состоит из следующих узлов: опорный остов (представляет собой дюралюминиевую плиту); два внешних и два внутренних борта; передняя и задняя стенки; электромеханический привод; электрооборудование (рис. 1).

Основными частями электромеханического привода являются два электромотора с понижающими редукторами, которые устанавливаются в левый и правый внутренние борта соответственно. Между электродвигателями и редукторами осуществляется ременная передача. В качестве ремней служат замкнутые растяжимые жгуты в количестве две единицы на приводную пару. Свойство растяжимости ремней позволяет в конструкции отказаться от натяжных механизмов и, как следствие, облегчить и упростить конструкцию. Так же растяжимость компенсирует резкое возрастание динамической нагрузки, предохраняя электродвигатель и редуктор от вредного, пагубного воздействия, тем самым увеличивает ресурс и срок эксплуатации модели. Но стоит отметить, что растяжимость ремней несколько снижает КПД и общую эффективность использования энергоресурсов.

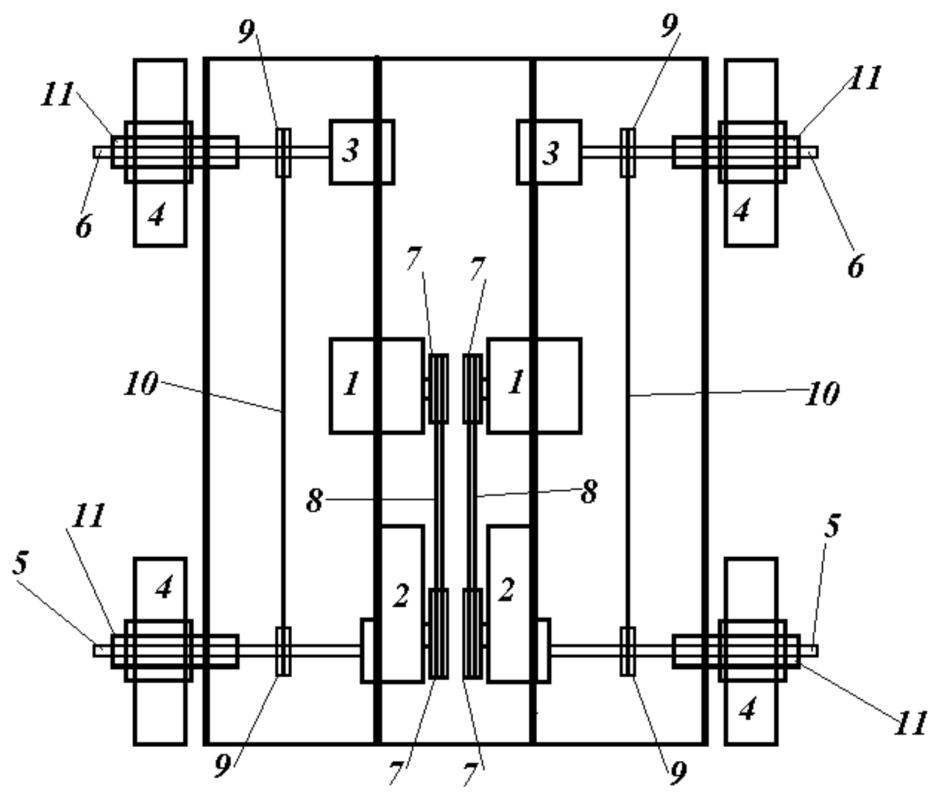

Рис. 1. Кинематическая схема: 1 - электродвигатель; 2 - понижающий редуктор;

3 - подпятник; 4 -колесо; 5 - ведущая полуось; 6-ведомая полуось; 7 - шкив; 8 -ременная передача; 9 - звёздочка; 10 - цепная передача; 11 - цапфа 
Во внешние борта монтируются стальные цапфы. Фиксируются при помощи резьбового соединения. На цапфы устанавливаются движители, являющиеся колесами диаметра 250 мм. Модель шасси робота - четырёхколёсная, имеет односкатную ошиновку.

Борта внутренние и внешние закрепляются на остове при помощи болтового соединения. С торцов на модель устанавливаются передняя и задняя стенки, которые придают конструкции дополнительную жёсткость и стойкость к динамическим нагрузкам. Стенки также предотвращают повреждение механизмов, расположенных внутри модели, от внешних воздействий.

Первая пара полуосей (ведущая), проходя через цапфы, взаимодействует с редуктором и колесом, осуществляя передачу крутящего момента и мощности. На полуоси устанавливаются ступицы, фиксируются болтовым соединением. На ступицы, при помощи точечной электросварки, закрепляются звездочки. Вторая пара полуосей (ведомая), аналогично первой, проходит через цапфы. Для фиксации второго конца ведомой полуоси необходима дополнительная деталь, так как опоры в виде редуктора отсутствуют. Подпятник выполняет функцию опоры, закрепляется на внешней стороне внутренних бортов. В подпятник устанавливается шариковый подшипник качения. Ведомая полуось взаимодействует с колесом и подпятником. На вторую пару полуосей также устанавливаются ступицы со звёздочками.

Каждый электродвигатель бортоповоротного шасси осуществляет приведение в движение модели со своей стороны, левой или правой. Управление происходит при помощи изменения частоты вращения электродвигателя. Благодаря такой схеме привода, модель является чрезвычайно маневренной и имеет возможность разворота на одном месте, что расширяет возможности, позволяет использовать модель в условиях замкнутого и ограниченного пространства. Модель робота имеет постоянный полный привод, независима от внешних источников питания, так как имеет на своём борту аккумуляторную батарею и может работать автономно.

Двигатель постоянного тока (ДПТ), который выбран в качестве электродвигателя шасси, находит широкое применение в различных областях деятельности, начиная от использования тягового привода в трамваях и троллейбусах, заканчивая приводом прокатных станов и подъемных механизмов, где требуется поддержание высокой точности скорости вращения.
В электродвигателях постоянного тока крутящий момент порождается реакцией между двумя магнитными полями: одно поле устанавливается стационарной обмоткой возбуждения, а другое - обмотками во вращающемся якоре. У некоторых двигателей постоянного тока отсутствует обмотка возбуждения, вместо которой установлены большие постоянные магниты, сохраняющие стационарное магнитное поле постоянным при любых рабочих условиях. В любом случае, принцип работы электродвигателя постоянного тока заключается в том, что ток, проходя через якорь, создает магнитное поле, пытающееся выровняться со стационарным полем, и поэтому якорь вращается [7].

Лучшим решением для управления оборотами двигателя постоянного тока является широтно-импульсная модуляция (ШИМ). В широком понимании ШИМ - это операция получения изменяющегося аналогового значения посредством цифровых устройств. Эти устройства используются для получения прямоугольных импульсов сигнала, который постоянно переключается между максимальным и минимальным значениями. Данный сигнал моделирует напряжение между максимальным значением (5 В) и минимальным (0 В), изменяя при этом длительность времени включения 5 В относительно включения 0 В. Длительность включения максимального значения называется шириной импульса. Для получения различных аналоговых величин изменяется ширина импульса.

На практике, в нашем случае, ШИМ регулятор был реализован с помощью микроконтроллера Arduino UNO. Коллекторные двигатели требуют гораздо большего тока, чем может выдавать встроенный блок питания Arduino UNO. Также они могут создавать опасные выбросы напряжения. Для управления ДПТ необходимо сделать гальваническую развязку между двигателем и контроллером, т.е. подключать его к отдельному источнику питания. Для этого ДПТ включается в коллекторную цепь биполярного транзисторного усилителя, который в свою очередь подключен к выходу ШИМ Arduino [8].

В программной среде ArduinoIDE была разработана программа автоматического управления скоростью вращения двигателя по желаемому графику (рис. 3, a). Также написана и проверена программа управления скоростью вращения вала с помощью потенциометра, подключаемого к одному из аналоговых входов Arduino UNO (рис. 3, б). Листинги фрагментов программ приведены на рис. 4. 


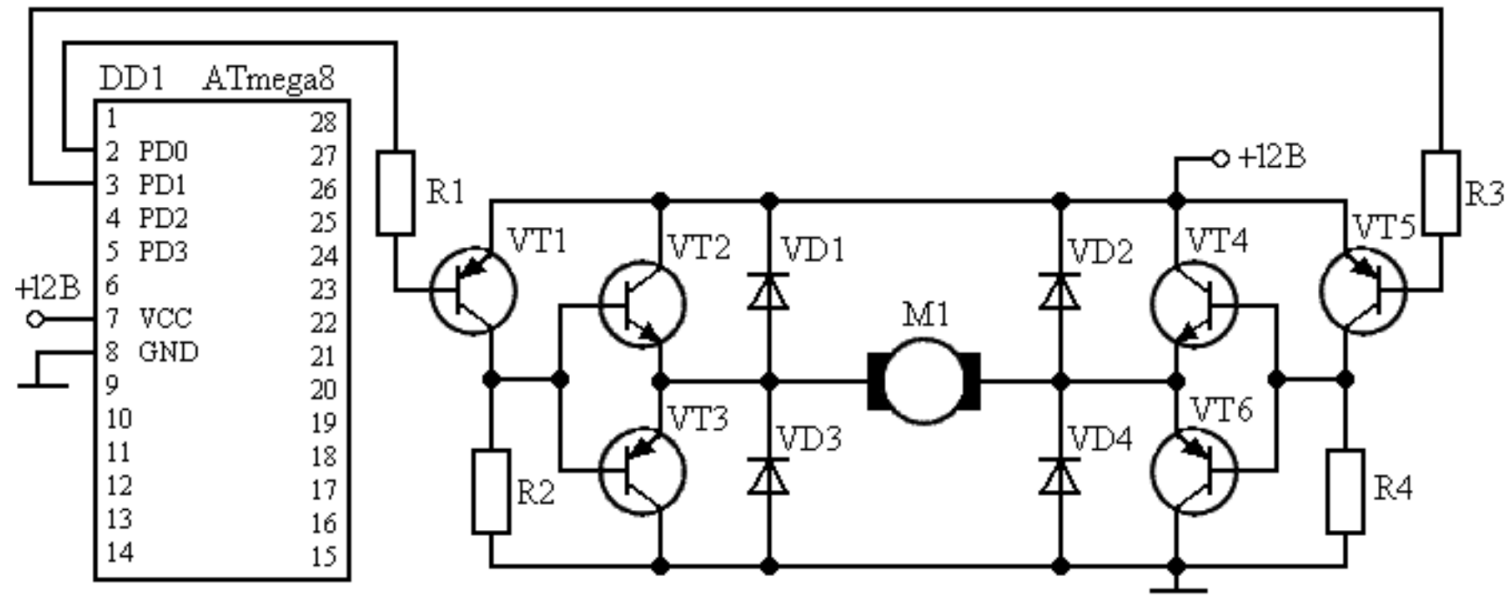

Рис. 2. Схема управления оборотами двигателя постоянного тока на базе микроконтроллера

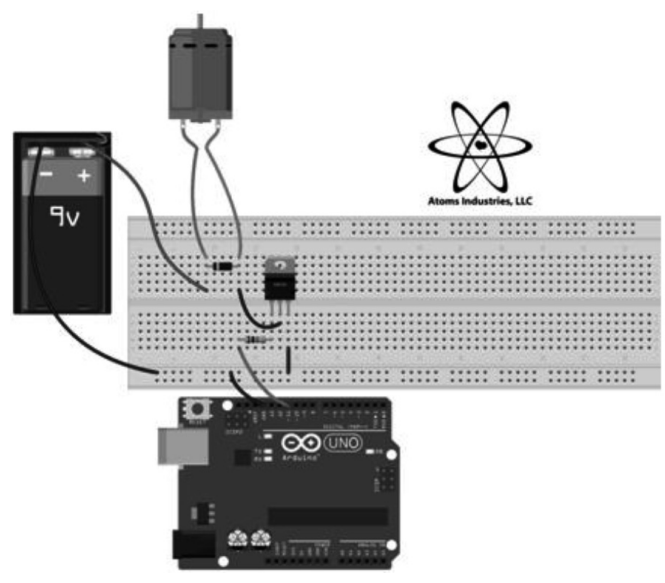

a)

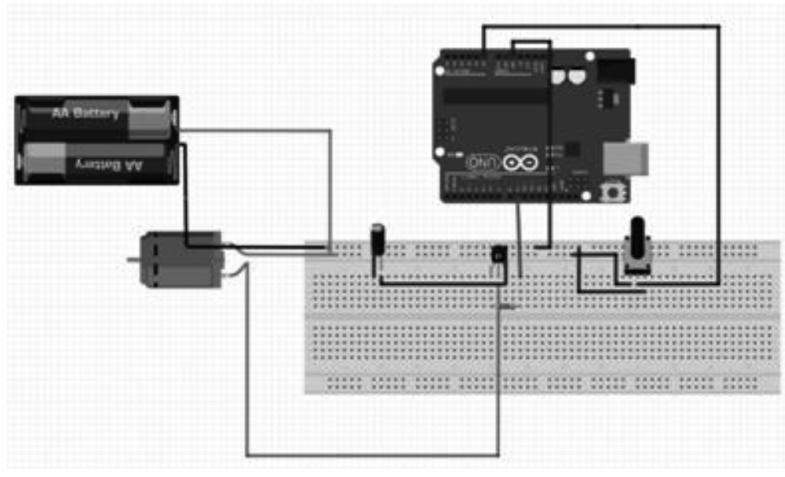

б)

Рис. 3. Электрическая цุепь управления оборотами двигателя постоянного тока на основе Аrduіпо: а) программное, б) потеничиометром

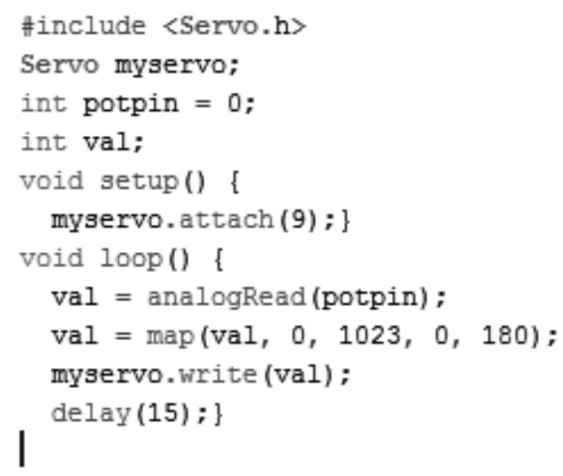

Рис. 4. Фрагментыл листинга программ

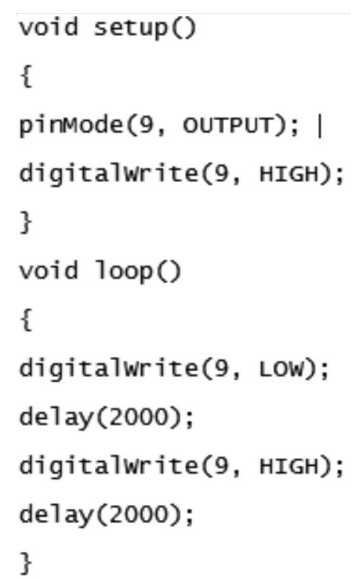


Следующим этапом в работе стал выбор системы навигации мобильного робота. Уже на сегодняшний день существует множество систем навигации с достаточно высокой точностью определения местоположения, однако каждая из них обладает своим набором плюсов и минусов. Это связано с развитием более глобальных навигационных методов, ненаправленных на ориентирование в условиях локальной местности, где необходимо повышенное внимание к точности [9-11].

После проведения анализа и теоретических исследований были выбраны следующие, как наиболее эффективные в имеющихся условиях, способы навигации: метод отражённой волны и инерциальный метод.

Первый рассматриваемый способ основан на акустическом излучении сигнала передатчиком. Скорость ультразвукового сигнала в воздухе является известной величиной, и в условиях локальной навигации можно её принимать за константу. Передатчик генерирует короткий ультразвуковой импульс около 40 кГц. При излучении данного сигнала происходит его отражение от ближайших объектов. Произведя замер времени задержки излученного сигнала, появляется возможность определения расстояния до объектов, отражающих излучённые сигналы. Данный метод не может быть использован в глобальной навигации, так как скорость звуковой волны на больших расстояниях различна.

Плюсами этого способа являются: простота конструкции, контроллер, не требующий большой вычислительной мощности, достаточно высокая точность определения расстояния до объектов. К недостаткам можно отнести невозможность определения точной координаты объекта навигации и высокое потребление электроэнергии, так как система работает в активном режиме.

Для улучшения точности было принято решение совместить этот способ с инерциальным датчиком ориентирования, т.е. с методом определения координат. Инерциальный метод основан на свойствах инерции тел и является автономным, т.е. не требующим наличия внешних ориентиров или поступающих извне сигналов [12-14].

Конечно, данный способ имеет как достоинства, так и недостатки. Среди плюсов можно выделить следующие параметры: автономность (способность работать независимо от других навигационных приборов); помехозащищённость (на прибор влияет только ускорение притяжения к Земле, тем самым обеспечивается защита от электромагнитных волн, способных сбить с толку остальные части общей системы навига- ции); быстродействие и точность (в некоторых случаях). Существенным их недостатком, не свойственным радиотехническим и оптико-электронным методам, является накопление ошибки измерения за время активной работы. Данная методическая погрешность обусловлена интегрирующим действием самой системы [15].

Для подтверждения преимуществ и недостатков выбранного метода навигации был разработан экспериментальный образец совмещенной системы. На базе микроконтроллера Arduino Uno был реализован метод отраженной волны. В качестве устройства приёма-передачи сигналов был выбран ультразвуковой дальномер НCSR04. Для увеличения угла обзора данного устройства был использован сервопривод, способный отклоняться на 180 градусов, приводя во вращение дальномер. Данная система способна обнаруживать объекты на расстоянии до 0,5 м и с точностью $\pm 0,01$ м. Для графического изображения на экране монитора был использован язык программирования Processing, благодаря которому есть возможность увидеть сам процесс ориентирования. Питание установки осуществляется с помощью кабеля USB от компьютера. В ходе испытаний был выявлен недостаток - малый радиус действия устройства, однако проблема может быть решена путём замены дальномера на более мощный датчик приёма-передачи.

При объединении данных двух методов навигации возникли проблемы, для решения которых были применены фильтры, выполняющие роль следящей системы, улучшающей точность посредством корректировки получаемых с датчиков данных. Первый фильтр - фильтр Маджвика. Он позволяет, опираясь на параллельно получаемые данные со встроенного в модуль магнитометра, с большой точностью определять углы поворота всей подвижной системы. Второй фильтр основан на использовании одного из ультразвуковых датчиков: датчик неподвижно закрепляется на коллинеарной линии с линией прямолинейного движения объекта и инерциального модуля по одной из осей координат $x$ или $y$ на выбор; программно, на основе получаемых данных с ультразвукового датчика, происходит процесс вычисления расстояния до ближайшего объекта и дальнейший расчёт разницы между начальным расстоянием до объекта и текущим; получив, таким образом, пройденное расстояние, оно сравнивается с данными пройденного пути, полученного от инерциального модуля, тем самым корректируя общее месторасположение. 


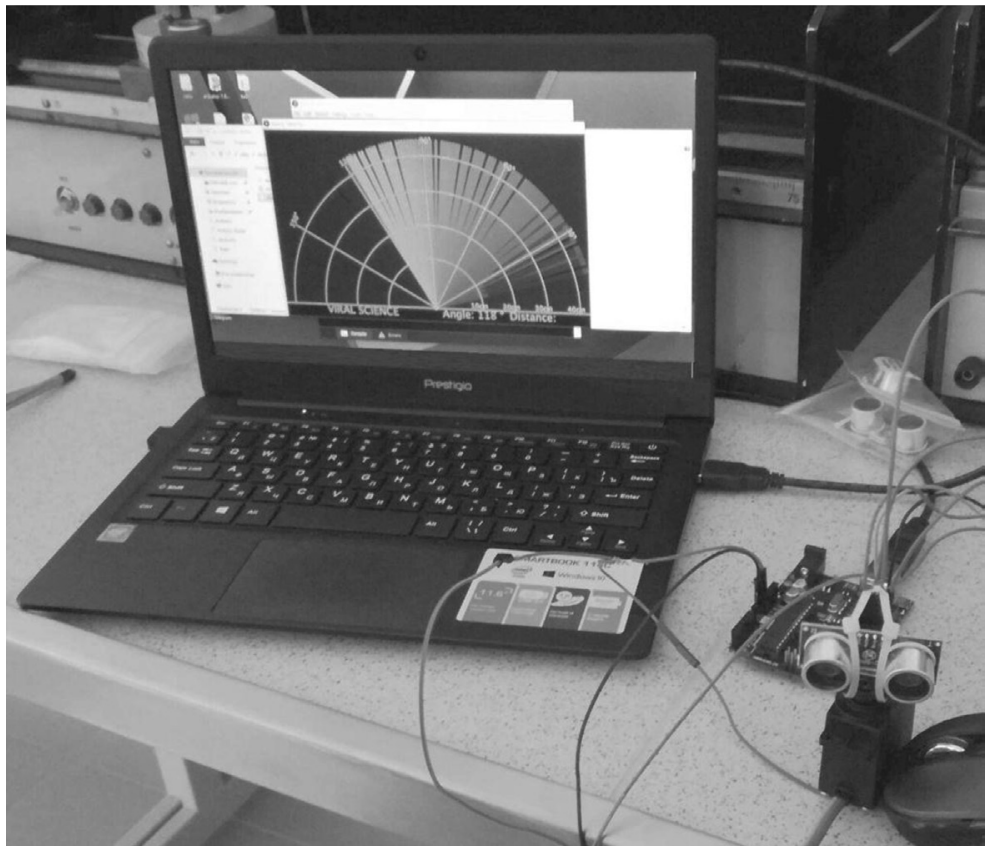

Рис. 5. Фотография установки волнового зондирования

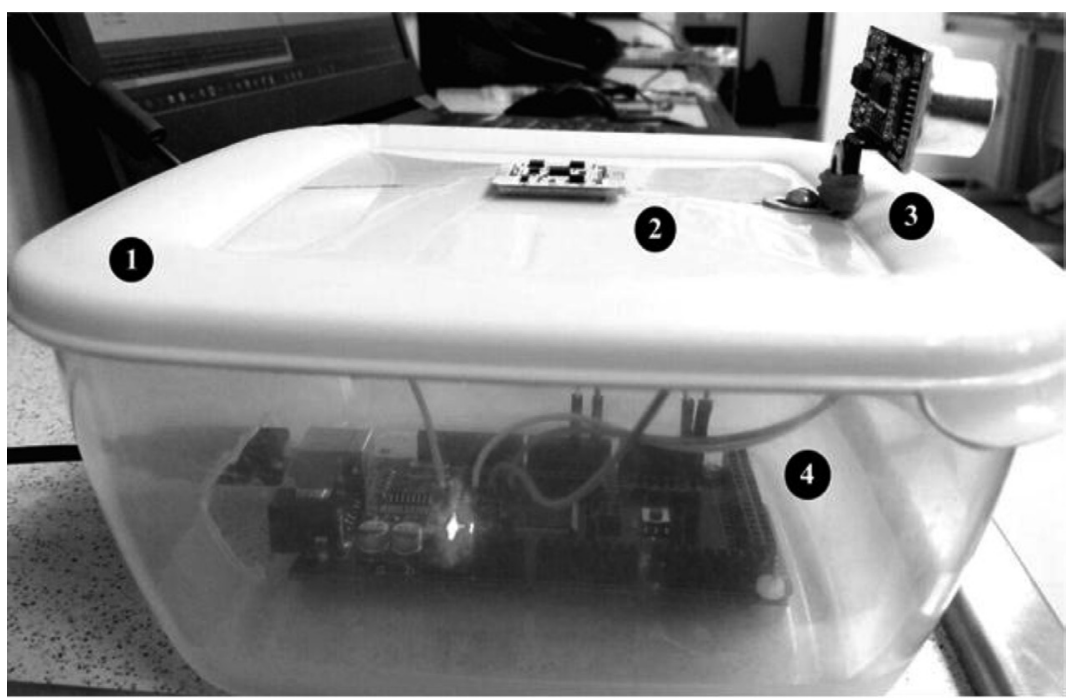

Рис. 6. Экспериментальная модель реализации интегрированной системь инерциильно-зондовой навигациии

На основе вышеизложенных замечаний, проанализированных преимуществах и недостатках различных методов, исходя из прошлых наработок, участниками СНК была создана модель для реализации интегрированной системы инерциально-зондовой навигации (рис. 6). Она состоит из контейнера (1), в котором была закреплена плата микроконтроллера Arduino Mega (4), инерциального модуля (2), состоящего из гироскопа, магнитометра, барометра и акселерометра, и ультразвукового модуля (3). Модули были закреплены на крышке контейнера неподвижно, а также расположены на одной линии, чтобы уменьшить вероятность технических погрешностей во время работы.

Все датчики работают одновременно и, благодаря мощной начинке Arduino Mega, не влияют друг на друга, тем самым позволяя без каких-либо проблем произвести интеграцию данных. Однако во время реализации интегрирования данных способов навигации друг с другом были выявлены следующие проблемы: 
- большой объём информации, который необходимо обрабатывать посредством микроконтроллера;

- возникновение на ранней стадии конфликта между реализуемыми системами ориентирования.

Несмотря на возникшие проблемы, которые можно решить при продолжении исследований, интеграция нескольких методов навигации на одной платформе дала очень хорошие результаты в плане точности ориентирования объекта в локальном пространстве. Даже при условии, что совмещение нескольких систем навигации привело к удорожанию экспериментальной модели, по сравнению с рыночными образцами она выглядит предпочтительнее.

\section{Заключение}

В результате научно-технической деятельности студенческого научного кружка «Робототехника, механотроника и электроника» было спроектировано и в лабораторных условиях разработано бортоповоротное шасси для экспериментальной модели мобильного робота. В дальнейшем планируется проведение экспериментов, результаты которых можно будет перенести, с некоторой адаптацией, на полноценные робототехнические системы. Использование микроконтроллера для изменения скорости вращения двигателя постоянного тока дает возможность точно регулировать скорость вращения вала без вмешательств в сам привод.

Также будут продолжены работы по устранению недостатков разработанной инерциально-зондовой системы навигации и по её улучшению в сторону удешевления и упрощения модели. Для этого будет дополнительно интегрирован фаза-частотный метод, который основан на формировании объектом навигации двух высокочастотных гармонических сигналов с разными частотами, их одновременном излучении с объекта навигации и приеме в нескольких опорных радионавигационных точках с известными координатами.

\section{Список литературы}

1. Егоров О.Д., Подураев Ю.В., Буйнов М.А. Робототехнические мехатронные системы. - М.: Станкин, 2015. - 326 с.

2. Ильясова Ф.С., Александров А.П. Робототехника: развитие, современное положение и перспективы // Информационно-компьютерные технологии в экономике, образовании и социальной сфере. - 2016. - № 1. - С. 85-95.

3. Юревич Е.И. Основы робототехники. - 2-е изд., перераб. и доп. - СПб.: БХВ-Петербург, 2005. - 401 с.

4. Горшунов А.А. Методы навигации с применением волнового зондирования // Материалы X Международной студенческой электронной научной конференции «Студенческий научный форум». URL: www.scienceforum. ru/2018/3000/3753 (дата обращения: 13.07.2018).

5. Кузьменко С.В. Разновидности инерциальной навигация и её дальнейшее развитие // Материалы X Международной студенческой электронной научной конференции «Студенческий научный форум». URL: www.scienceforum. $\mathrm{ru} / 2018 / 3000 / 3750$ (дата обращения: 13.07.2018).

6. Сергеев Е.И. Применение компьютерного зрения при ориентировании в пространстве // Материалы X Международной студенческой электронной научной конференции «Студенческий научный форум». URL: www.scienceforum. ru/2018/3000/3754 (дата обращения: 13.07.2018).

7. Джоган В.А. Электродвигатель постоянного тока // Энергия будущего: материалы научно-практической конференции студентов электроэнергетического факультета. 2018. - C. 33-37.

8. Боряк С.В., Куприянов И.В. Регулирование скорости вращения двигателя постоянного тока с применением ПИДрегулятора на основе микроконтроллера Arduino Uno // Вестник науки и образования. - 2016. - № 4 (16). - С. 21-23.

9. Гриценко А.В., Дорошенко Н.С. Исследование и классификация методов распознавания изображения в системах компьютерного зрения // Вестник Ставропольского государственного университета. - 2011. - № 4. - С. 84-89.

10. Селетков И.П., Машкин С.В. Построение 3d-модели среды по видео изображению с некалиброванной камеры в режиме реального времени // Вестник Пермского университета. Серия: Информационные системы и технологии. 2011. - № 12. - С. 80-85.

11. Семенов А.Е., Крюков Е.В., Рыкованов Д.П., Семенов Д.А. Практическое применение технологий компьютерного зрения в решении задач распознавания, восстановления $3 \mathrm{~d}$, сшивки карт, точного целеуказания, счисления пути и навигашии // Известия ЮФУ. Технические науки . $2010 .-$ № 3 (104). - C. 92-102.

12. Мелешко В.В., Нестеренко О.И. Бесплатформенные инерциальные навигационные системы: учебное пособие. Кировоград, 2011.- $171 \mathrm{c}$

13. Соловьев В.И., Шабалов П.Г. Инерциальные навигационные системы: учебное пособие. - Самара: Изд-во СГАУ, 2011. - $71 \mathrm{c}$.

14. Шевцова Е.В. Погрешности инерциальных навигационных систем: учебное пособие. - М.: Изд-во МГТУ им Н.Э. Баумана, 2012. -24 с.

15. Ломанов А.Н., Ревунов В.В. Сравнительный обзор аэронавигашионных методов автоматического счисления пути // Вестник Рыбинской государственной авиационной технологической академии им. П.А. Соловьева. - 2016. № 1 (36). - C. 108-115. 